Article

\title{
Overweight or Obesity Onset and Past Attempts to Lose/Manage Weight: A Qualitative Study
}

\author{
Ziaul Hasan Rana ${ }^{1, *}$, Debra B. Reed ${ }^{1}$, Wilna Oldewage-Theron ${ }^{1}{ }^{\mathbb{D}}$, Conrad Lyford ${ }^{2}$, Malinda Colwell ${ }^{3}$ \\ and John A. Dawson ${ }^{1}$ \\ 1 Department of Nutritional Sciences, Texas Tech University, 2500 Broadway, Lubbock, TX 79409, USA; \\ debra.reed@ttu.edu (D.B.R.); wilna.oldewage@ttu.edu (W.O.-T.); john.a.dawson@ttu.edu (J.A.D.) \\ 2 Department of Agricultural and Applied Economics, Texas Tech University, 2500 Broadway, \\ Lubbock, TX 79409, USA; conrad.lyford@ttu.edu \\ 3 Department of Human Development and Family Studies, Texas Tech University, 2500 Broadway, \\ Lubbock, TX 79409, USA; malinda.colwell@ttu.edu \\ * Correspondence: zhranadu@gmail.com; Tel.: +1-(806)-500-9335
}

Citation: Rana, Z.H.; Reed, D.B.; Oldewage-Theron, W.; Lyford, C.; Colwell, M.; Dawson, J.A. Overweight or Obesity Onset and Past Attempts to Lose/Manage Weight: A Qualitative Study. Obesities 2021, 1, 136-143. https://doi.org/ 10.3390/Obesities1030012

Academic Editor: Nobuyuki Takahashi

Received: 2 August 2021

Accepted: 1 October 2021

Published: 6 October 2021

Publisher's Note: MDPI stays neutral with regard to jurisdictional claims in published maps and institutional affiliations.

Copyright: (C) 2021 by the authors. Licensee MDPI, Basel, Switzerland. This article is an open access article distributed under the terms and conditions of the Creative Commons Attribution (CC BY) license (https:/ / creativecommons.org/licenses/by/ $4.0 /)$.

\begin{abstract}
This study aims to investigate the prevalence of weight control attempts along with their strategies and motivations. This study used a retrospective observational qualitative method, and adults who had body mass index (BMI) of at least $25 \mathrm{~kg} / \mathrm{m}^{2}$ were included. The qualitative data were analyzed using a descriptive phenomenological approach. The summaries and emergence of major concepts identified by the participants were analyzed in-depth using a systematized retrieval and review of the data. NVivo was used to establish interrater reliability and percent agreement and analyze and manage the data. $91.7 \%$ of participants had tried to lose weight, and $72 \%$ were successful at least in one of their attempts. Health, appearance, and sports training were salient motivating factors. Their attempts to lose weight ranged from 2 to 30 times (median 4 times). Different dietary strategies and physical exercises were often tried. Among the most notable strategies were diet methods, following weight management programs, and consulting with dietitians. A number of barriers were reported, including ineffective weight loss, non-sustainability, demotivation, and unaffordability. For advancing evidence-based long-term weight control, it is vital to evaluate the quality and nature of weight management strategies and services from the perspective of users.
\end{abstract}

Keywords: obesity; overweight; weight management; diet; physical activity; qualitative

\section{Introduction}

Obesity is a chronic disease, and successful treatment requires deep-rooted lifelong effort. Weight management requires a proper assessment and management, and this assessment includes determining its degree of overweight or obesity and general health status [1,2]. Weight reduction is associated with reduced blood pressure, lowered blood glucose, and improved dyslipidemias that are related to obesity [1,3]. Weight control efforts by adults play an important role in public health because they help people manage their weight by following evidence-based and effective weight management methods [2]. Furthermore, the patient's willingness to make major lifestyle changes must also be considered before participating in treatment for weight loss. Studies recommend that the assessment of weight loss status should incorporate the reasons and inspiration for weight loss, results of past endeavors at weight reduction, support expected from family and companions, comprehension of dangers and advantages, attitudes toward physical activity, time accessibility, and potential challenges, including money-related limitations and the patient's reception of progress [1,4].

The relationship between personal weight-loss attempts and obesity is complex, and well-informed efforts to get in shape may result in more effective weight loss and maintenance [5]. Evidence suggests that among overweight and obese individuals, the number of 
weight-loss attempts is a negative indicator of weight-loss progress [6]. Recurrent weight control may negatively affect body image, self-perception, pessimistic attributions, and feelings of weakness, which can lead to disappointment and failure [7]. In order to provide a better understanding of what individuals look for in a weight control program as well as what they are currently doing and why, it is essential to learn what is the number of weight control endeavors made, by whom, why, and how. A study showed $36 \%$ of general practice patients had tried to lose weight at least once in the past twelve months, with a large number of patients of normal weight practicing weight loss [4]. The most prevalent method of losing weight was dieting, regardless of whether they practiced exercise and/or workout [8]. Although many examinations have been performed in the past, they provide little insight into the explicit kinds of weight-loss procedures by overweight or obese individuals, including whether they consulted their general practitioners before they changed their eating habits or weight-loss strategies [9,10]. A better understanding of this information can aid in improving weight reduction interventions. In light of these factors, the purpose of this study is to assess the prevalence of personal weight control attempts and to distinguish respondents and their personal strategies used, fundamental thinking processes, and underlying motivations. Using the results of this study will provide better insight into weight management strategies and the weight reduction discussion.

\section{Materials and Methods}

\subsection{Participants, Recruitment, Ethics, and Consent}

This study used a retrospective observational method. The study included individuals age 18 and older with a BMI of 25 or more. Based upon a review of the literature regarding phenomenological approaches, a sample size of 12 was sought [11]. Interested participants were recruited in response to the university announcement, including 7 men and 5 women. Interested individuals were phone-screened to determine whether they met the eligibility criteria when they contacted researchers. The study was conducted in Lubbock, in the northwest region of Texas. All procedures in this study conformed to the regulations of the University Institutional Review Board. Written informed participation consent was sought and obtained for all participants upon enrollment in the study. Participation in this study required a personal visit to the clinical study lab on the university campus to obtain informed consent and complete the questionnaire. Participants' heights and weights were measured after obtaining informed consent. The height was measured with a stadiometer and the weight with a digital TANITA health scale.

\subsection{Data Collection}

After an individual met the inclusion criteria, a semi-structured interview was scheduled. During the interview, participants were encouraged to describe their weight loss or weight maintenance experiences as thoroughly as possible. After a close-ended question, participants had the opportunity to provide detailed responses to any of the open-ended questions. Particularly, the last section was to write down in detail all the experiences they had regarding weight loss or maintenance. The research questions addressed in this study were: (1) How many times have individuals with BMI $\geq 25$ tried to lose weight? (2) What motivation(s) work when trying to lose weight? (3) What strategies were used to lose/manage weight? (3) What are the potential barriers when trying to lose or manage weight? Therefore, the measurable variables of this study were the timing of the first diagnostics and present situation regarding weight management, attempts and strategies to lose weight, specific goals during weight-loss attempts, and strategies that were used during successful attempts. The primary investigator read and reread the interview, extracting significant statements that captured the essence of the interview [12].

\subsection{Statistical Analysis}

We followed a systematized retrieval and review of data to distinguish summary concepts and their relationships. Data were analyzed in qualitative terms using Colaizzi's 
descriptive phenomenological framework [13]. To gain an understanding of the experiences of participants, two investigators independently read and reread the transcripts. Then, they extracted statements that described the phenomenon and illuminated the experience. Based on these statements, the researchers formulated meanings and assigned these meanings codes, words, or phrases that describe and summarize the meaning of the subject's experience. Codes were organized into themes, represented by categories of experiences that were universal to all participants, and unified into a comprehensive description of the phenomenon. Detailed discussion was developed until a consensus was reached, and the trustworthiness of the data was enhanced by the transparent and straightforward process of analysis. Credibility was established by having two of the study team members analyze the data independently, followed by a third member with expertise in qualitative methodology validating the themes and coding. NVivo was used for qualitative data analysis and management and to establish two measures of interrater reliability and percent agreement. Descriptive statistics were calculated using IBM SPSS (Statistics for Windows, Version 23.0).

\section{Results}

A total of 12 participants were recruited and surveyed for this study (Table 1). Seven of them were men. The participants ranged in age from 18 to 52 years, with a median age of 23 years. The youngest was 19, and the oldest was 52 . Among the participants, $41.7 \%$ were currently trying to lose weight. Another $41.7 \%$ of participants were not actively trying to lose weight, but they were aware of their high BMI. $8.3 \%$ of the participants tried to gain weight, while another $8.3 \%$ wanted to maintain their weight. $90 \%$ of the participants had attempted to lose weight prior to the study. Losing weight is attempted by participants between 2 and 30 times, with a median attempt of 4 . Among those who tried to lose weight in the past, $72 \%$ were successful during at least one attempt, and the rest failed every time.

Table 1. Weight loss/maintenance status of the participants.

\begin{tabular}{lc}
\hline Variables & N (\%) or Median \\
\hline Number of participants & 12 \\
\hline Age (Median) & 23 \\
\hline Sex & $7(58.3)$ \\
\hline Male & $5(41.7)$ \\
\hline Female & \\
\hline Current situation of weight management & $5(41.7)$ \\
\hline Trying to lose weight & $5(41.7)$ \\
\hline$\quad$ Not actively trying to do anything & $1(8.3)$ \\
\hline Trying to gain weight & $1(8.3)$ \\
\hline Want to maintain same weight & $11(91.7)$ \\
\hline Tried to lose weight in the past & 4 \\
\hline Number of times they attempted to lose weight (Median) & $8(71.2)$ \\
\hline Successful in at least one of their attempts
\end{tabular}

\subsection{Strategies Applied to Lose or Manage Weight}

Participants who try to lose or manage weight implemented various strategies that they believed would enable them to lose or manage weight. They did workouts, for example, with different cardio or heart stimulating exercises, running, stepping, and playing different sports such as American football. One of the participants mentioned, "The strategy I like the most was exercise-induced. I overloaded my schedule with sports so I was working out at least twice a day with my team. I was not losing weight but I was not gaining either which 
was good for me." Some of them practiced on more than one occasion each week. Some of them described one of their weight-loss systems as becoming busy at home with their daily activities. Moreover, other weight management programs were followed, for example, Jenny Craig or Nutrisystem. One participant mentioned, "The 3rd time was Nutri system [sic], just because I wanted to lose some weight".

Moreover, supervision from a dietitian or nutritionist was also an attempted strategy. A participant stated, "American football team nutritionist strictly controlled (through access) diet and weight training program [sic]." They additionally adjusted their weight control plans by different diet and exercise strategies, for example, skipping meals, eating smaller portions, and reducing carbohydrate-rich foods. One of the participants stated, "My most successful weight-loss strategies were when I was playing football, because I was working out or running 5 days a week. My diet consisted of high protein and low fat." Moreover, a couple responded they had followed the Ketogenic diet, which included very low amounts of carbohydrates with a high amount of protein and fat. One said, "Now I am thinking about KETO. My doctor is after me to lose the weight and I am unable to get on the ride at 6 flags [sic]".

\subsection{Dieting Strategies}

Some participants believed that the concept of dieting was associated with the number of calories and types of food that they consumed. People who followed some diet regimes generally measured the caloric intake and amount of food and believed that their eating regimen had to be adjusted. They believed in order to lose weight, they should focus on food containing low amounts of fat and sugar and a high amount of protein. They held the idea of abstaining from excessive food intake and limited eating. They also believed dieting required skipping meals. "Once I start monitoring my food intake and amount of time exercising. I always begin to feel better. My problem is the consistency to keep that going. Writing things down and keeping a daily log seemed to be most beneficial" quoted by a participant.

\subsection{Weight Management Programs and Dietitian}

Different weight management programs and dietitian or doctor supervision were also followed. Some of the respondents had a better outcome with these programs and supervision. For example, they learned to eat healthier foods and drink more water. They also learned the nutrition facts of different foods, and the specific guidelines provided were more beneficial. A participant said, "My 2nd time was the supervision of a doctor (dietitian). She gave me a special food program based on my daily, and more commonly usual foods, for example, I used to eat banana, potato. She was not forcing me to eat special food which even I did not regularly use it. We had weekly checkup which helps me a lot to continue my effort and not to stop it. This session was good motivation to pay attention to my everyday food schedule. She put me a lot of water drink before the meal, which made you feel full before each meal. Water have zero calories and give no desire to have more food. In 7 month, I lost 15 to $17 \mathrm{~kg}$ ". Another participant stated, "My first diet was Jenny Craig, I lost a lot of weight that state off about a year."

\subsection{Duration of Weight Loss Attempts}

The study participants who attempted to lose weight were doing this for a long time, ranging from months to years. One participant mentioned, "American football team nutritionist strictly controlled (through access) diet and weight training program for three years". In some cases, respondents attempted certain strategies for a couple of weeks, suspended them, and moved to different techniques. One participant quoted, "Until I could lose some weight." Some participants tried for a fixed amount of time by involving some tasks or programs. One said, "Self-directed exercise and eating program while in the wilderness for 2 months." However, some subjects did not follow the traditional strategies paying little mind to exercise, watching calories, dieting, or any professional programs. 


\subsection{Motivations to Lose Weight}

The participants shared their experiences and mentioned several elements that motivated them to apply various weight-loss strategies. Health, appearance, social support, and sports training were motivating factors in their previous weight-loss attempts. The aim to be physically sound and be more attractive was mentioned when the participants were asked about their motivation to lose weight. One said, "My doctor is after me to lose the weight and I am unable to get on the ride at 6 flags [sic]". Furthermore, some of them encountered medical issues and trusted that losing weight might enhance their conditions. A vital condition was the support from close persons, for example, companions, significant others, and other relatives. A participant mentioned, "My father used to count my calories and monitor my food intake".

\subsection{Unsustainability and Ineffectiveness}

Some of them stated their dissatisfaction when they were not able to lose weight despite their endeavors to utilize different techniques, including exercise. The weight reduction was unsustainable, and they regained weight once they halted the practice or suspended the program. "About 5 years ago, I did a strict low carb diet and lost about $50 \mathrm{lbs}$. I went back to eating a normal diet and gained it all back plus 30 more pounds. I have made several more attempts with low carb, but have not had those same results," said a participant. A few of them asserted that they regained more weight than the initial weight they started with after adopting a weight-loss strategy. At the end of the day, their weight after taking part in a strategy was the same or higher than before starting the strategies.

\subsection{Lack of Self-Control \& Demotivation}

The most widely recognized confinements to an effective weight reduction were simply lack of self-control and inspiration. A big number of the participants utilized words, such as "absence of control", "absence of order", and irregularity. One of them quoted "No strategy was really successful. In the best cases, I could not just keep the weight. But I also keep the weight more or less constant even when I am not trying to keep or lose it". Additionally, the impacts of weight-loss strategies to get thinner are viewed as basic factors that decide if they would conform to the picked strategies. Participants also became demotivated when the strategies did not deliver the expected impacts.

\subsection{Unaffordability of Strategies}

Some of the participants used weight management programs as mentioned previously or took continuous consultation from dietitians. Even though they mentioned they had the desired results for a certain period, they could not continue for a long time due to the expense. A participant stated, "The 3rd time I was Nutri system [sic], just because I wanted to lose some weight. I lost $20 \mathrm{lbs}$, but it was expensive. So I quit".

\section{Discussion}

Public health emphasizes the importance of weight control efforts, and it is essential to understand why, how, and who attempts weight loss in order to provide a more detailed picture of this phenomenon. Our exploratory study was therefore designed to determine how often individuals attempt to control their weight, as well as to describe respondents strategies and their motivations behind managing their weight. The study found that different approaches to weight loss were often adapted through different diet and exercise plans. Different weight management programs were also followed, and consultation with a dietitian was a notable strategy. Some participants counted occupation-related activities and home chores as forms of exercise. In addition, counting calories, reducing carbohydrates, skipping meals, and consuming smaller portions of food were practiced as well.

Investigating the idea of dieting, the respondents had certain knowledge and perceptions identified with weight reduction. A portrayal of dieting as consuming healthy food 
was also found in some similar studies [14,15]. The knowledge they had was mostly related to the consumption of calories, portion control, and meal moderation; few were concerned with exercising and other sports. Despite different health programs concentrating on losing weight to avoid chronic diseases, a portion of the overweight or obese respondents had never tried to lose weight, and some of them were content with their present weight status. A study in Europe showed that more than 40 percent of individuals who were overweight and obese did not have any intention to control their weight, and this lack of intent was more prevalent among males [16]. Besides, participants in this study expected a rapid response to their efforts. When they did not shed a substantial amount of weight, they became dissatisfied easily. They reported participating in a specific weight-loss strategy for a short period of time.

The majority of the subjects utilized different strategies to get more fit, which was likewise noted among different populaces from various studies. An Australian study on attempts to lose weight found that participants used a variety of procedures and techniques to lose or maintain a desirable weight [17]. Participants often mentioned that they wanted to be healthy and fit as well as to have a better physical appearance through losing weight. The most dominant motivation for people living with overweight or obesity was to be healthier. Those who are overweight or obese and suffering from cardiovascular disease or hypertension are more likely to want to lose weight [18]. Physical appearance was equally important to both genders [19].

Weight-loss efforts tend to fail because of overeating habits, a preference for unhealthy foods, and a lack of persistence in exercise. In a USA study, those who skipped breakfast and ate more breakfast or supper in restaurants were more likely to become overweight or obese [20]. The fat and cholesterol content of restaurant food is higher than that of homemade food [20]. Additionally, the environment and biological dispositions play a critical role in weight gain and weight loss [21]. People, such as life partners, relatives, companions, and others in the immediate environment, serve as both constructive and antagonistic factors in weight loss and management efforts [19].

To achieve the desired weight, most participants lacked motivation and self-discipline to maintain good dietary habits and exercise consistency. Different studies have characterized these challenges as the most common [22]. By focusing on simple approaches, participants sought an easy way to lose weight. When their weight-loss strategies failed or they regained the weight they had lost, participants became discouraged. The ineffectiveness and non-sustainability to lose weight and encounter of weight cycling were common constraints. In a study of African American women with pre-obesity or obesity, nearly two-thirds experienced weight cycling when they lost 20 pounds, then regained it [23]. These cycles were characterized by binge eating and body image disturbances. Despite studies indicating that past weight-loss attempts may adversely influence current weight-loss results, individuals who have tried multiple times may lack self-confidence and self-reliance and, as a result, succeed less in future attempts [6,24]. The global and national objectives should be to promote a healthy way of reducing weight and preventing chronic illnesses in addition to educating people about appropriate weight-loss strategies.

The presented results should be seen considering the weaknesses and strengths of the study. The primary limitation was that the study included participants residing in Texas, USA; therefore, highlighting a need for further research on the relation between perceived overweight and weight management in other regional contexts. Moreover, although important potential moderators of weight control attempts were assessed, other variables with potential moderator effects were not assessed, such as socioeconomic status or education level. The sample size was also relatively small. A larger sample would provide a strong statistical strength. Additionally, weight control motives, which are typically influenced by social desirability or desirability, were assessed among a small number of participants, likely leading to over or underestimated results. Despite some weaknesses in the present study, the benefits of the study on attempts to lose weight and weight control practices should not be underestimated. As a major strength of this 
study, a wide array of personal weight control strategies was assessed using a qualitative approach. A real-life case study of obese and overweight individuals who lost weight in the past was investigated in this study. Further, this study revealed the major obstacles and barriers to achieving a satisfactory result. Thus, the findings provided relevant insights to ensure the acceptability, sustainability, and effectiveness of weight-loss intervention programs. Importantly, the study opened up new possibilities for further research and improvement of current health promotion strategies.

\section{Conclusions}

This study investigated the key strategies and motives associated with weight loss/ management attempts and successes and challenges from the viewpoint of those actively living with overweight and obesity. Losing or managing weight often takes substantial investments of time, energy, and expectations, sometimes lasting years. The call for more weight loss education will be ineffective without providers who work with such patients being aware of the challenges of losing and keeping weight off. Considering the high demand for weight loss/management solutions, it is crucial to thoroughly evaluate the nature and quality of weight management strategies and services from the perspective of clients for advancing evidence-based and safe services and products targeting long-term weight control. This study outcome provided applicable experiences to guarantee the appropriateness and viability of a weight-loss intervention program and a premise to assist future experiments and improvements for current weight management procedures. People who are dealing with weight loss or maintenance wanted to talk about their weight-loss histories and positive and negative experiences. Therefore, more qualitative research should be set up so that we can listen when they want to talk. The conception of the idea of dieting needs to be adjusted, and the right concepts need to be embraced into food habits. Different scientifically demonstrated and easily applicable weight-loss techniques need to be explained, and sensible and realistic expectations and outcomes should be built up.

Author Contributions: Conceptualization, Z.H.R. and J.A.D.; methodology, Z.H.R., D.B.R., W.O.-T. and J.A.D.; software, Z.H.R. and J.A.D.; validation, D.B.R., W.O.-T., C.L. and M.C.; formal analysis, Z.H.R.; investigation, D.B.R., W.O.-T. and J.A.D.; writing—original draft preparation, Z.H.R., J.A.D.; writing - review and editing, Z.H.R., D.B.R., W.O.-T., C.L., M.C. and J.A.D.; supervision, J.A.D.; All authors have read and agreed to the published version of the manuscript.

Funding: The study was supported by Texas Tech University Institutional funding.

Institutional Review Board Statement: The study was conducted according to the guidelines of the Declaration of Helsinki, and approved by the Institutional Review Board of Texas Tech University.

Informed Consent Statement: Informed consent was obtained from all subjects involved in the study.

Data Availability Statement: The datasets used and/or analyzed during the current study and additional information are available from the corresponding author on reasonable request.

Acknowledgments: The authors would like to extend their gratitude to the local communities who aided in the data collection.

Conflicts of Interest: The authors declare no conflict of interest, financial or otherwise.

\section{References}

1. Wing, R.R.; Lang, W.; Wadden, T.A.; Safford, M.; Knowler, W.C.; Bertoni, A.G.; Hill, J.O.; Brancati, F.L.; Peters, A.; Wagenknecht, L. Benefits of modest weight loss in improving cardiovascular risk factors in overweight and obese individuals with type 2 diabetes. Am. Diabetes Care 2011, 34, 1481-1486. [CrossRef] [PubMed]

2. Raynor, H.A.; Champagne, C.M. Position of the Academy of Nutrition and Dietetics: Interventions for the Treatment of Overweight and Obesity in Adults. J. Acad. Nutr. Diet. 2016, 116, 129-147. [CrossRef] [PubMed]

3. Hales, C.M.; Carroll, M.D.; Fryar, C.D.; Ogden, C.L. Prevalence of Obesity Among Adults and Youth: United States, 2015-2016. NCHS Data Brief. 2017, 288, 1-8.

4. Santos, I.; Sniehotta, F.F.; Marques, M.M.; Carraça, E.V.; Teixeira, P.J. Prevalence of personal weight control attempts in adults: A systematic review and meta-analysis. Obes. Rev. 2017, 18, 32-50. [CrossRef] 
5. Teixeira, P.J.; Going, S.B.; Sardinha, L.B.; Lohman, T.G. A review of psychosocial pre-treatment predictors of weight control. Obes. Rev. 2005, 6, 43-65. [CrossRef] [PubMed]

6. Strohacker, K.; Carpenter, K.C.; McFarlin, B.K. Consequences of Weight Cycling: An Increase in Disease Risk? Int. J. Exerc. Sci. 2009, 2, 191-201. [PubMed]

7. Kruger, J.; Galuska, D.A.; Serdula, M.K.; Jones, D.A. Attempting to lose weight. Am. J. Prev. Med. 2004, 26, 402-406. [CrossRef]

8. Leavy, J.M.; Clifton, P.M.; Keogh, J.B. The role of choice in weight loss strategies: A systematic review and meta-analysis. Nutrients 2018, 10, 1136. [CrossRef]

9. Ismail, T.A.T.; Jalil, R.A.; Wan Ishak, W.R.; Hamid, N.F.; Nik, W.S.W.; Mohamed, H.J.J.; Mohd, N.H.; Arifin, W.N.; Mohamed, W.M.I.W.; Ibrahim, M.I.; et al. Understanding dieting and previous weight loss attempts among overweight and obese participants: Insights into my body is fit and fabulous at work program. Korean J. Fam. Med. 2018, 39, 15. [CrossRef]

10. Yoong, S.L.; Carey, M.L.; Sanson-Fisher, R.W.; Deste, C. A cross-sectional study assessing the self-reported weight loss strategies used by adult Australian general practice patients. BMC Fam. Pract. 2012, 13, 1-7. [CrossRef]

11. Vasileiou, K.; Barnett, J.; Thorpe, S.; Young, T. Characterising and justifying sample size sufficiency in interview-based studies: Systematic analysis of qualitative health research over a 15-year period. BMC Med. Res. Methodol. 2018, 23, 53-74. [CrossRef] [PubMed]

12. Murimi, M.W.; Kanyi, M.G.; Mbogori, T.N.; Amin, M.R.; Rana, Z.H.; Nguyen, B.; Moyeda-Carabaza, A.F. Voices and perspectives of rural Hispanic households on food insecurity in west Texas: A qualitative study. J. Hunger Environ. Nutr. 2019, 14, 540-557. [CrossRef]

13. Abalos, E.E.; Rivera, R.Y.; Locsin, R.C.; Schoenhofer, S.O. Husserlian Phenomenology and Colaizzi's Method of Data Analysis: Exemplar in Qualitative Nursing Inquiry Using Nursing as Caring Theory. Int. J. Hum. Caring 2016, 20, 19-23. [CrossRef]

14. Sweeting, H.; Smith, E.; Neary, J.; Wright, C. "Now I care": A qualitative study of how overweight adolescents managed their weight in the transition to adulthood. BMJ Open 2016, 6, e010774. [CrossRef] [PubMed]

15. Bisogni, C.A.; Jastran, M.; Seligson, M.; Thompson, A. How People Interpret Healthy Eating: Contributions of Qualitative Research. J. Nutr. Educ. Behav. 2012, 44, 282-301. [CrossRef]

16. Santos, I.; Andrade, A.M.; Teixeira, P.J. Prevalence of weight control attempts and behavioral strategies among Portuguese adults: A cross-national survey. Obes. Facts 2013, 28, 77-86.

17. Thomas, S.L.; Hyde, J.; Karunaratne, A.; Kausman, R.; Komesaroff, P.A. “They all work...when you stick to them": A qualitative investigation of dieting, weight loss, and physical exercise, in obese individuals. Nutr. J. 2008, 7, 1-7. [CrossRef]

18. Piernas, C.; Aveyard, P.; Jebb, S.A. Recent trends in weight loss attempts: Repeated cross-sectional analyses from the health survey for England. Int. J. Obes. 2016, 40, 1754-1759. [CrossRef]

19. Teixeira, P.J.; Silva, M.N.; Mata, J.; Palmeira, A.L.; Markland, D. Motivation, self-determination, and long-term weight control. Int. J. Behav. Nutr. Phys. Act. 2012, 9, 22. [CrossRef]

20. Ma, Y.; Bertone, E.R.; Stanek, E.J.; Reed, G.W.; Hebert, J.R.; Cohen, N.L.; Merriam, P.A.; Ockene, I.S. Association between eating patterns and obesity in a free-living US adult population. Am. J. Epidemiol. 2003, 57, 1552-1560. [CrossRef]

21. Sharifi, N.; Mahdavi, R.; Ebrahimi-Mameghani, M. Perceived Barriers to Weight loss Programs for Overweight or Obese Women. Heal. Promot. Perspect. 2013, 3, 11. [CrossRef]

22. Alnasser, A.A.; Alkhalifa, A.S.; Sathiaseelan, A.; Marais, D. What Overweight Women Want from a Weight Loss App: A Qualitative Study on Arabic Women. JMIR mHealth uHealth 2015, 3, e4409. [CrossRef] [PubMed]

23. Osborn, R.L.; Forys, K.L.; Psota, T.L.; Sbrocco, T. Yo-Yo dieting in African American women: Weight cycling and health. Ethn. Dis. 2011, 21, 274-280. [PubMed]

24. Myers, V.H.; McVay, M.A.; Champagne, C.M.; Hollis, J.F.; Coughlin, J.W.; Funk, K.L.; Gullion, C.M.; Jerome, G.J.; Loria, C.M.; Samuel-Hodge, C.D.; et al. Weight loss history as a predictor of weight loss: Results from Phase I of the weight loss maintenance trial. J. Behav. Med. 2013, 36, 574-582. [CrossRef] [PubMed] 